\title{
miR-34a inhibits cell proliferation in prostate cancer by downregulation of SIRT1 expression
}

\author{
KUN DUAN $^{1 *}$, YONG-CHAO GE $^{1 *}$, XUE-PEI ZHANG ${ }^{2}$, SHU-YI WU ${ }^{3}$, JIN-SHUN FENG $^{1}$, \\ SHI-LIN CHEN ${ }^{1}$, LI ZHANG ${ }^{1}$, ZHI-HAO YUAN ${ }^{1}$ and CHAO-HONG FU ${ }^{1}$
}

\author{
${ }^{1}$ Department of Urology Surgery, The Third People's Hospital of Zhengzhou; ${ }^{2}$ Department of Urology Surgery, \\ The First Affiliated Hospital of Zhengzhou University; ${ }^{3}$ Department of Stem Cell and Organ Transplantation Laboratory, \\ The Third People's Hospital of Zhengzhou, Zhengzhou, Henan, P.R. China
}

Received October 19, 2014; Accepted July 16, 2015

DOI: $10.3892 / 01.2015 .3645$

\begin{abstract}
MicroRNA-34a (miR-34a) functions as a tumor suppressor gene and inhibits abnormal cell growth by regulating the expression of other genes. The role of miR-34a in regulating sirtuin 1 (SIRT1) in prostate cancer remains unclear. The objective of the present study was to investigate the biological function and molecular mechanisms of miR-34a regulation of SIRT1 in human prostate cancer samples and the human prostate cancer cell line, PC-3. Fresh prostate tissues were obtained from patients, and the miR-34a expression in prostate cancer tissues was measured using reverse transcription-quantitative polymerase chain reaction ( $\mathrm{RT}$-qPCR). qPCR and western blotting were performed to assess the effects of miR-34a overexpression on SIRT1 regulation in PC-3 cells, and the cell growth was assessed by Cell Counting Kit-8 (CCK-8). Flow cytometry was used to assess the cell cycle status of the cells. The miR-34a expression levels in prostate cancer tissues were significantly reduced compared with adjacent normal prostate tissues $(\mathrm{P}<0.05)$. SIRT1 expression levels in PC-3 cells with over-expression of miR-34a were significantly reduced compared with those in the negative control $(\mathrm{P}<0.05)$. The over-expression of miR-34a inhibited PC-3 cells growth and resulted in increased cell cycle arrest compared with the negative control $(\mathrm{P}<0.05)$. In conclusion, miR-34a inhibits the human prostate cancer cell proliferation, in part, through the downregulation of SIRT1 expression.
\end{abstract}

Correspondence to: Professor Yong-Chao Ge, Department of Urology Surgery, The Third People's Hospital of Zhengzhou, 136 South Street, Guancheng Hui, Zhengzhou, Henan 450000, P.R. China

E-mail: bgx1980@163.com

${ }^{*}$ Contributed equally

Key words: sirtuin 1, microRNA-34a, human prostate cancer cell, cell proliferation

\section{Introduction}

Prostate cancer is the second most common urological malignancy and the sixth leading cause of cancer-associated mortality in men (1). Based on GLOBOCAN 2012 estimates, 1.1 million new cases occurred worldwide in 2012, accounting for $\sim 307,000$ deaths (1). Previous studies indicated that miRNA expression may be used as an optimal strategy for predicting the therapeutic outcome of prostate cancer $(2,3)$. Therefore, the interference of cancer-specific miRNAs could be exploited to produce a direct anticancer effect.

MicroRNAs (miRs) are short non-coding RNAs that bind to the 3' untranslated region (UTR) of target mRNAs and are involved in post-transcriptional control of gene expression by inhibiting translation (4). miRNAs are also considered to target $>50 \%$ of all human genes (5). Accumulating evidence indicate that miRNAs are important regulatory molecules in various biological processes, including cell differentiation, proliferation, apoptosis and metabolism (6). miR-34a is located on chromosome 1p-36.23 in humans and highly expressed in multiple types of cancer such as colon cancer and lung cancer (7). The tumor suppressor protein p53 induces transcription of miR-34a and the expression of miR-34a correlates well with activation of p53 by genotoxic stress activation (8). A previous study, using non-transfected miR-34a mimics as the negative control, demonstrated that increased expression levels of miR-34a were associated with wild-type p53 tumors expressing reduced Bcl-2 levels compared with in tissues with reduced miR-34a expression (9). Therefore, the role of miR-34a as a tumor suppressive RNA may be p53 or p53-pathway dependent. The ectopic expression of miR-34a induces arrest of the cell cycle in G1 phase, apoptosis and senescence in tumors. Moreover, a number of target mRNAs of miR-34a have been determined including CDK4/6, cyclin D1, $c$-Met and E2F3 (10).

Sirtuin 1 (SIRT1), a NAD ${ }^{+}$-dependent class III histone deacetylase, is involved in a wide range of cellular processes including cell proliferation, senescence and apoptosis, by affecting DNA repair, stress response and aging (11). It has been indicated that SIRT1-defective or knockdown cells have an increased apoptotic response to DNA damage or oxidative stress treatments (12). Although numerous studies have 
indicated a role for SIRT1 in tumorigenesis, its role in cancer has not been sufficiently studied. For example, SIRT1 demonstrates anti-oncogene action in colon cancer and its expression level is associated with prognosis, but it is considered to exhibit oncogenic action in breast cancer $(13,14)$. A previous study indicated that tumors in SIRT1-deficient mice have markedly increased numbers of cells undergoing apoptosis (15). Moreover, elevated expression levels of SIRT1 have been observed in several types of human malignancies, including ovarian, liver, stomach, ductal, and pancreatic cancers (16). Additionally, previous studies have demonstrated that SIRT1 regulated various molecules, including p-53, FOXO1-4, NF-kB, hypermethylated in cancer 1 (HIC1) and E2F1 $(17,18)$. However, the regulatory control of SIRT1 in prostate cancer remains unclear.

The present study investigated the biological function and molecular mechanisms of miR-34a regulation of SIRT1 in human prostate cancer samples. In addition, the regulation of miR-34a by SIRT1 and its potential molecular mechanisms in prostate cancer were investigated by transfecting an miR-34a inhibitor into human prostate cancer PC-3 cells.

\section{Materials and methods}

Tissue samples. Patient-matched prostate cancer and normal prostate tissues (15 pairs) were obtained from patients who underwent radical prostatectomy at the Department of Urology, the Third People's Hospital of Zhengzhou (Zhengzhou, China). Prostate cancer tissue specimens $(n=15)$ were identified as prostatic adenocarcinoma. None of the cases had received any previous cancer-associated treatment, or had a history of any other types of cancer. All patients underwent pre-treatment evaluation, including bone scan, chest X-ray, and magnetic resonance imaging (MRI) of the abdomen and pelvis to evaluate the tumor stage. Prostate cancer stage was classified according to the seventh American Joint Committee on Cancer (AJCC) system (19). All the samples were snap-frozen in liquid nitrogen immediately and stored at $-80^{\circ} \mathrm{C}$ following surgery, until RNA extraction. The histological diagnosis was confirmed by examining hematoxylin and eosin-stained original sections simultaneously by two pathologists. The study protocol was approved by the Local Ethics Committees of Zhengzhou University, and written informed consent was obtained from all patients prior to tissue collection.

Cell culture and transfection. The prostate cancer PC-3 cell line was purchased from the Cell Resource Center of Shanghai Institutes for Biological Sciences, Chinese Academy of Sciences (Shanghai, China). PC-3 cells were cultured in RPMI-1640 medium containing $10 \%$ fetal bovine serum (FBS) and $100 \mathrm{U} / \mathrm{ml}$ penicillin/streptomycin (Invitrogen Life Technologies, Carlsbad, CA, USA) in a humidified atmosphere of $5 \% \mathrm{CO}_{2}$ maintained at $37^{\circ} \mathrm{C}$. The PC-3 cells were seeded at a density of $1 \times 10^{5}$ cells per well in 6 -well plates and transfected with $50 \mathrm{nM}$ of miR-34a mimics or the negative control (GenePharma, Co., Ltd., Shanghai, China) using Lipofectamine ${ }^{\circledR}$ RNAiMAX (Invitrogen Life Technologies) according to the manufacturer's instructions. The cells were then collected $48 \mathrm{~h}$ after transfection.
RNA extraction and reverse transcription-quantitative polymerase chain reaction $(R T-q P C R)$. Total RNA from fresh prostate tissues and cultured PC-3 cells was isolated using TRIzol reagent (Omega Bio-Tek, Norcross, GA, USA) according to the manufacturer's instructions. RNA quantity and quality were determined using $1.0 \%$ agarose gel electrophoresis and an optical density 260/280 absorption ratio of $>1.8$ using the NanoDrop 2000 (Thermo Fisher Scientific, Waltham, MA, USA). The High Capacity cDNA reverse transcription kit for RT-PCR ${ }^{\circledR}$ (Takara Bio, Inc., Otsu, Japan) was used for the synthesis of $20 \mu \mathrm{l}$ complementary DNA (cDNA) from 1,000 ng of whole RNA.

mRNA expression levels were determined by RT-qPCR, which were measured by the ABI PRISM 7300 Sequence Detection System using the SYBR ${ }^{\circledR}$ Green PCR Master mix (Applied Biosystems, Foster City, CA, USA). Cycling conditions were as follows: initial activation at $50^{\circ} \mathrm{C}$ for $2 \mathrm{~min}$, and denaturation at $95^{\circ} \mathrm{C}$ for $10 \mathrm{~min}$, followed by 40 cycles of $95^{\circ} \mathrm{C}$ for $15 \mathrm{sec}$ and $60^{\circ} \mathrm{C}$ for $1 \mathrm{~min}$. The expression of the house-keeping gene, $18 \mathrm{~s}$ rRNA, as an internal control was examined under the same reaction conditions. The experiment was conducted in triplicate. Gene expression was quantified in relation to the values of the control group following normalization against the internal control using the $2^{-\Delta \Delta C}$ method. The following RT-qPCR oligonucleotide primers were used: Forward, 5'-TGGCAGTGTCTTAGCT-3' and reverse, 5'-TGGTGTCGTGGAGTCG-3' for miR34a; forward, 5'-CCCAGAACATAGACACGCTGGA-3' and reverse, 5'-ATCAGCTGGGCACCTAGGACA-3' for SIRT1; and forward, 5'-TTCGGAACTGAGGCCATGAT-3' and reverse, 5'-CGAACCTCCGACTTTCGTTT-3' for 18S rRNA.

Cell proliferation analysis. The PC-3 cells were transferred to 96-well microplates at 1,000 cells/well $24 \mathrm{~h}$ after transfection. The effects of miR-34a on cell proliferation were detected 0,24 and $48 \mathrm{~h}$ after seeding using the Cell Counting Kit- 8 (CCK-8; Dojindo Molecular Technologies, Kumamoto, Japan) according to the manufacturer's instructions. Each assay was replicated 5 times.

Flow cytometry cell cycle analysis. PC-3 cells were transfected with miR-34a for $48 \mathrm{~h}$, as descibed above. The cells were then collected, and washed with cold phosphate-buffered saline (PBS) containing 1\% FBS, fixed in cold $70 \%$ ethanol in PBS for at least $24 \mathrm{~h}$ and stained with propidium iodide (Sigma-Aldrich, St. Louis, MO, USA). Following staining, the cell cycle statuses were determined by FACSCalibur (Becton Dickinson, Mountain View, CA, USA) using CellQuest Pro software (BD Biosciences, Franklin Lakes, NJ, USA). The cell cycle fractions were analyzed by ModFit software 3.0 (Verity Software House, Inc.).

Western blot analysis. Total protein was extracted from the cells using a cell lysis buffer and protease inhibitor mixture (Beyotime Institute of Biotechnology, Jiangsu, China). After centrifugation at $10,000 \times \mathrm{g}$ and $4^{\circ} \mathrm{C}$ for $15 \mathrm{~min}$, the protein concentration was determined by the Bradford protein assay kit (Bio-Rad Laboratories, Hercules, CA, USA), according to the manufacturer's instructions. The sample loading buffer was added to the protein sample and heated at $100^{\circ} \mathrm{C}$ 


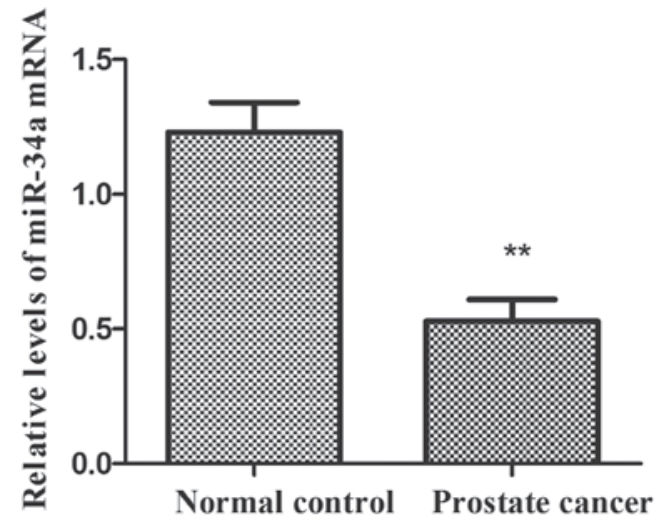

Figure 1. Relative expression of miR-34a in prostate cancer tissues and normal prostate tissues were examined by RT-qPCR. The data demonstrates the mRNA expression levels of miR-34a in prostate cancer relative to the normal prostate tissues ( $\mathrm{n}=15,{ }^{* *} \mathrm{P}<0.001$, compared with normal control). All the experiments were performed 3 times independently.

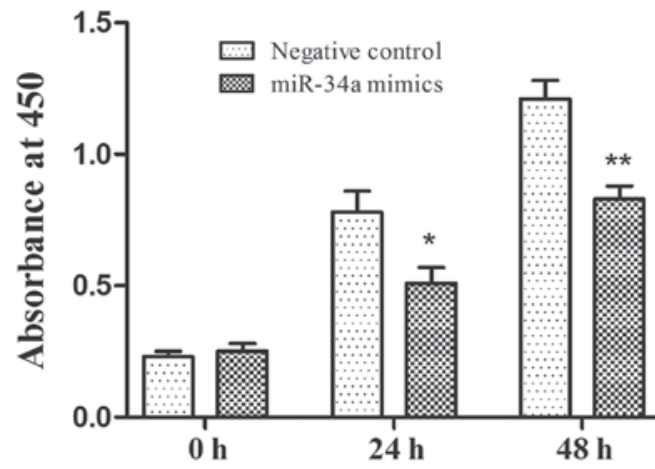

Figure 2. The effects of miR-34a on growth of prostate cancer cell lines. Suppressive effects of miR-34a on the growth of PC-3 cells were observed. After transfection $(24 \mathrm{~h})$, cells were seeded into 96-well plates at a density of 1,000 cells/well. The effect of miR-34a on cell proliferation was detected at 0,24 and $48 \mathrm{~h}$ after seeding using Cell Counting Kit- $8\left(\mathrm{n}=4,{ }^{*} \mathrm{P}<0.05\right.$ and ${ }^{* * *} \mathrm{P}<0.01$, compared with the negative control).

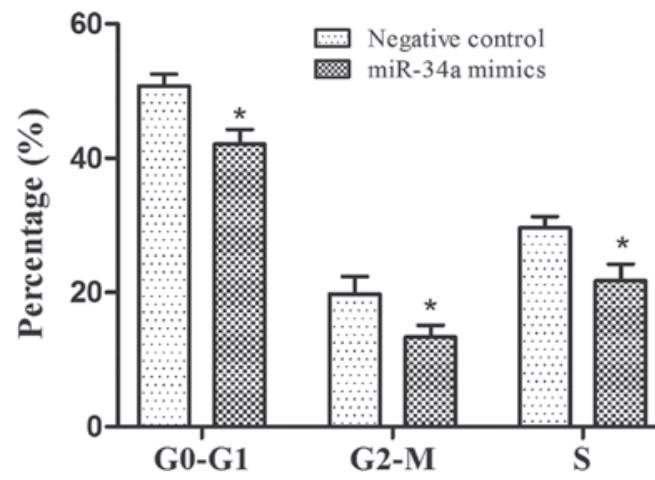

Figure 3. The effect of miR-34a on cell cycle distribution in PC-3 cell lines. PC-3 cells were extracted at $48 \mathrm{~h}$ post-transfection. PC- 3 cells transfected with miR-34a mimics compared with the negative control demonstrated that the cell cycle was significantly arrested in the miR-34a mimics groups $(n=4$, ${ }^{*} \mathrm{P}<0.05$, compared with the negative control).

for $10 \mathrm{~min}$. The proteins $(20 \mu \mathrm{g})$ were then loaded onto a $12 \%$ SDS-PAGE gel and transferred onto polyvinylidene difluoride (PVDF) membranes (Amersham Biosciences, Piscataway, NJ, USA). The PVDF membranes were blocked using 5\% non-fat dry milk in a Tris-buffered sodium chloride-Tween-20 (TBST) solution at room temperature for $1 \mathrm{~h}$, and incubated with monoclonal rabbit anti-SIRT1 $(1: 1,000$; Abcam, Cambridge, MA, USA) overnight at $4^{\circ} \mathrm{C}$. After washing, the membranes were incubated with horseradish peroxidase-labeled secondary anti-rabbit antibody (1:2,000; Abcam) at room temperature for $2 \mathrm{~h}$. Following three $10-\mathrm{min}$ washes in TBST, the immunoreactive bands were detected using western blot chemiluminescence luminol reagents (Santa Cruz Biotechnology, Inc., Santa Cruz CA, USA). The band intensities were quantified using scanning densitometry (Bio-Rad Quantity One software; Bio-Rad).

Statistical analysis. Data are presented as the mean \pm standard deviation. The differences were analyzed using the Student's $t$-test. All the analyses were performed using SPSS software, version 17.0 (SPSS Inc., Chicago, IL, USA). P<0.05 was considered to indicate a statistically significant difference.

\section{Results}

miR-34a expression in prostate cancer. miR-34a expression levels in human prostate cancer tissues and adjacent normal prostate tissues were examined by RT-qPCR (Fig. 1). The expression levels of miR-34a were significantly reduced in human prostate cancer tissues compared with the adjacent normal prostate tissues $(\mathrm{P}<0.01)$. The results indicated that the downregulation of miR-34a may be involved in human prostate carcinogenesis.

The effect of miR-34a on cell proliferation and cycle progression in human prostate cancer $\mathrm{PC}-3$ cell. To investigate the antiproliferative function of miR-34a in prostate cancer cells, human prostate cancer PC-3 cells were transfected with miR-34a mimics and the negative control. CCK-8 was used to examine the proliferation rate of $\mathrm{PC}-3$ cell $24 \mathrm{~h}$ after transfection and over the following 3 days. Compared with the negative control, the overexpression of miR-34a significantly inhibited the cell proliferation rate in the PC-3 cell lines (Fig. 2; $\mathrm{P}<0.05$ ). The effect of miR-34a on cell cycle progression was examined by flow cytometry. The percentage of PC-3 cells in S phase in the miR-34a mimics group was increased compared with the negative control $(\mathrm{P}<0.05)$, and there was a significant reduction in the proportion of cells arrested in the G0-G1 phase and G2-M phase in the miR-34a mimics group compared with negative control (Fig. 3) $(\mathrm{P}<0.05)$.

Expression levels of the miR-34a target gene, SIRT1, in PC-3 cells. The total RNA samples was isolated from PC-3 cells. The mRNA expression levels of SIRT1 following miR-34A transfection was examined by RT- qPCR. The results demonstrated that the mRNA expression levels of SIRT1 in the PC-3 cell line were significantly reduced in the miR-34a mimics group compared with the negative control group (Fig. 4; $\mathrm{P}<0.01$ ).

Western blot analysis was performed to analyze the expression levels of SIRT1 proteins in PC-3 cells. The bands of SIRT1 proteins were observed in miR-34a mimics and negative control groups. As was observed in SIRT1 mRNA levels, the protein expression levels of SIRT1 were reduced following miR-34a overexpression in PC-3 cells (Fig. 5A and B; $\mathrm{P}<0.05$ ). 


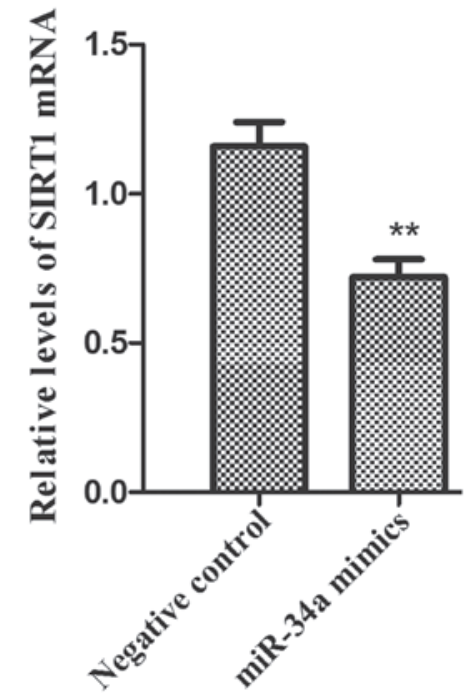

Figure 4. RT-qPCR analysis of SIRT1 mRNA expression in the PC-3 cells transfected with miR-34a. PC-3 cells were extracted at $48 \mathrm{~h}$ post-transfection. Relative mRNA expression of SIRT1 in PC-3 cells transfected with miR-34a mimics or the negative control. ( $\mathrm{n}=4,{ }^{* *} \mathrm{P}<0.01$ vs. negative control).
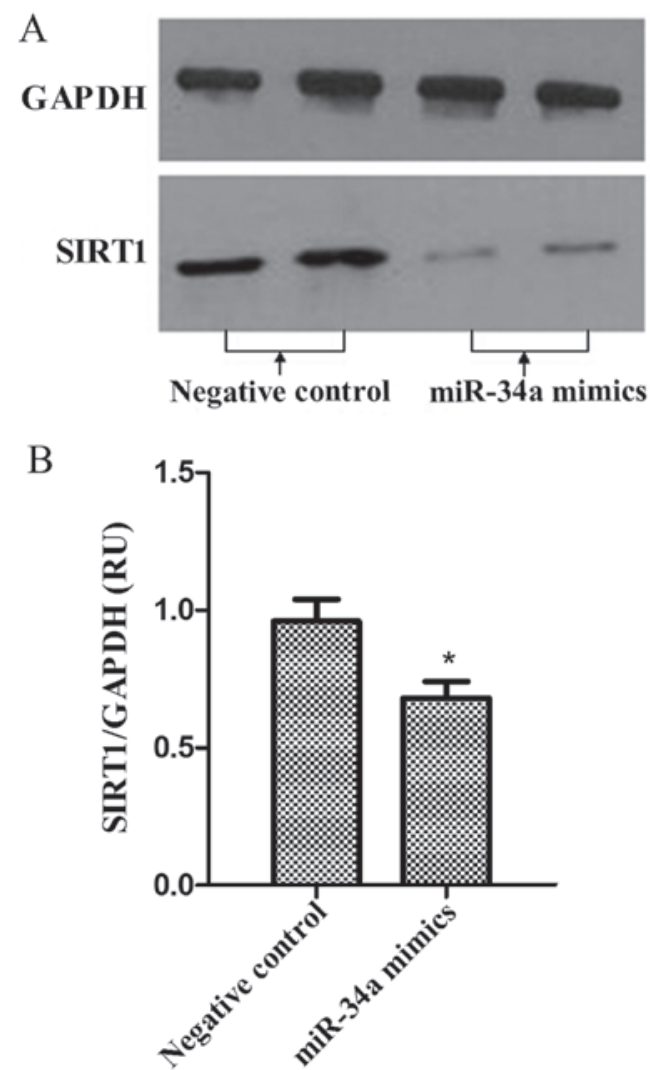

Figure 5. Western blot analysis of SIRT1 expression in the PC-3 cells transfected with miR-34a. Protein lysates were extracted at $48 \mathrm{~h}$ post-transfection. (A) Equal amount of protein was loaded into each well as indicated by the expression of the GAPDH protein. (B) Densitometry quantification of effect of transfection of miR-34a in the PC-3 cells ( $n=4, \pm$ SD; ${ }^{*} \mathrm{P}<0.05$ vs. negative control).

\section{Discussion}

A previous study reported that a low expression of miR-34a in certain types of human tumor was observed (20). In the present study, the results indicated that the expression of miR-34a was reduced in human prostate cancer tissues compared with the adjacent normal tissues. These results indicated that dysregulation of miR-34a may be involved in the pathogenesis of human prostate cancer. miR-34a is located on chromosome 1p-36; deletion or loss of heterozygosity of this region is associated with a number of types of human tumor, including breast cancer, lung cancer and cervical cancer (7). In addition, miR-34a is the target gene of the p53 tumor suppressor gene, and p53 tumor suppressor gene mutation is involved in the development of prostate cancer (21). $\mathrm{CpG}$ methylation is currently recognized as one of the mechanisms in carcinogenesis. miR-34a represents a tumor suppressor gene which is inactivated by $\mathrm{CpG}$ methylation and subsequent transcriptional silencing in various types of cancer including bladder cancer (22). Therefore, the downregulation of miR-34a expression may be of tumorgenesis in human prostate cancer.

SIRT1 has been demonstrated to protect cells against stress-induced senescence, which has been reported to be one of the potential targets of miR-34a (23). miR-34a is a recognized cancer cell inhibitor, was revealed as a posttranscriptional regulator of SIRT1. Moreover, SIRT1 is also involved in tumorigenesis, which is overexpressed in a number of types of human tumor, including colon, renal and lung cancer (24). In the present study, overexpression of miR-34a was observed to reduce the expression levels of SIRT1 compared with the negative control. miR-34a was overexpressed in endothelial progenitor cells (EPCs), and SIRT1 protein level was found to be diminished, which indicated that miR-34a negatively regulated SIRT1 expression in EPCs (25). Akao et al (26) reported that overexpression of miR-34a activated acetylation of p53 by mitigating SIRT1 activation in human colon cancer cells. However, in another study, overexpression of miR-34a did not completely suppress SIRT1 translation (23). These inconsistent results may be associated with the observation that miR-34a does not saturate its SIRT1 binding site or every SIRT1 binding site does not interact with miR-34a, so that a number of SIRT1 mRNA are still translated.

In the present study, the proliferation rate of human prostate cancer cells in miR-34a mimics group was significantly reduced compared with the negative control, which indicated that miR-34a may possess a significant antitumor effect on prostate cancer cells. The results were similar to observations in colon, breast and lung cancer, in which miR-34a expression was downregulated, and overexpression of miR-34a inhibited cell proliferation $(7,26,27)$. The present study selected SIRT1, an energy sensor, to validate the antitumor mechanism of miR-34a in prostate cancer cells. The downregulation of SIRT1 by miR-34a is considered to be part of a positive feedback loop acting on p53. Chapman et al (28) proposed that SIRT1 is involved in metabolism and tolerance to oxidative stress, promoting the growth of human urothelial cancer cell. Moreover, inhibition of SIRT1 expression reduced cell proliferation even in p53 mutated cells (29). The present study demonstrated that overexpression of miR-34a significantly reduced SIRT1 mRNA and protein expression levels. The results indicated that the proliferation inhibitory effect of miR-34a on human prostate cancer cell may be partly implemented by downregulating SIRT1 expression.

A previous study reported that miR-34a could induce the cell cycle arrest in tumors, especially in cell proliferation (30). 
In the present study, downregulation by miR-34a of SIRT1 was detected in human prostate cancer PC-3 cells. Certain proteins involved in cell cycle control have been identified as direct targets of miR-34a, including SIRT1, NMYC, MET and E2F3 (31). miR34-a induce cell cycle arrest via $\mathrm{p}-53$-miR-34a-SIRT1 axis was also observed in cancer cells and umbilical vein endothelial cells (32). In addition, the expression of SIRT1 mRNA was also significantly reduced in response to overexpression miR-34a in the present study: The results indicated that SIRT1 expression level may be regulated by miR-34a at the transcriptional and/or post-transcriptional levels. However, Yamakuchi et al (23) reported that overexpression of miR-34a in HCT116 human colon carcinoma cells downregulated SIRT1 protein expression, but did not affect its mRNA level (23). These inconsistent results indicate that the binding characteristics of miR-34a to the target gene sequence and its effects on gene regulation may vary depending on the cell lines.

In conclusion, the expression of miR-34a was reduced in prostate cancer tissues compared with the adjacent normal prostate tissues. The target gene SIRT1 of miR-34a was downregulated at the protein and mRNA levels when the expression of miR-34a was elevated in the human prostate cancer cells. The results of the present study indicated that miR-34a may inhibit carcinogenesis by downregulating the expression of SIRT1, therefore inhibiting cell proliferation and inducing cell cycle arrest in the human prostate cancer cells. Therefore, modulation of miR-34a activity may represent a novel approach for treating prostate cancer.

\section{Acknowledgements}

The present study was supported by grants from the key scientific and technological project of Henan province (no. 201004022).

\section{References}

1. Ferlay J, Soerjomataram I, Dikshit R, Eser S, Mathers C, Rebelo M, Parkin DM, Forman D and Bray F: Cancer incidence and mortality worldwide: Sources, methods, and major patterns in GLOBOCAN 2012. Int J Cancer 136: E359-E386, 2015.

2. Gade TP, Hassen W, Santos E, Gunset G, Saudemont A, Gong MC, Brentjens R, Zhong XS, Stephan M, Stefanski J, et al: Targeted elimination of prostate cancer by genetically directed human T lymphocytes. Cancer Res 65: 9080-9088, 2005.

3. Salter KH, Acharya CR, Walters KS, Redman R, Anguiano A, Garman KS, Anders CK, Mukherjee S, Dressman HK, Barry WT, et al: An integrated approach to the prediction of chemotherapeutic response in patients with breast cancer. PLoS One 3: e1908, 2008.

4. Denli AM, Tops BB, Plasterk RH, Ketting RF and Hannon GJ: Processing of primary microRNAs by the Microprocessor complex. Nature 432: 231-235, 2004.

5. Huntzinger E and Izaurralde E: Gene silencing by microRNAs: Contributions of translational repression and mRNA decay. Nat Rev Genet 12: 99-110, 2011.

6. Kloosterman WP and Plasterk RH: The diverse functions of microRNAs in animal development and disease. Dev Cell 11: $441-450,2006$

7. Li XJ, Ren ZJ and Tang JH: MicroRNA-34a: A potential therapeutic target in human cancer. Cell Death Dis 5: e1327, 2014.

8. Guessous F, Zhang Y, Kofman A, Catania A, Li Y, Schiff D, Purow B and Abounader R: MicroRNA-34a is tumor suppressive in brain tumors and glioma stem cells. Cell Cycle 9: 1031-1036, 2010.

9. Ji Q, Hao X, Meng Y, Zhang M, Desano J, Fan D and Xu L: Restoration of tumor suppressor miR-34 inhibits human p53-mutant gastric cancer tumorspheres. BMC Cancer 8: 266, 2008.
10. Maroof H, Salajegheh A, Smith RA and Lam AK: Role of microRNA-34 family in cancer with particular reference to cancer angiogenesis. Exp Mol Pathol 97: 298-304, 2014.

11. Satoh A, Stein L and Imai S: The role of mammalian sirtuins in the regulation of metabolism, aging and longevity. Handb Exp Pharmacol 206: 125-162, 2011.

12. Kabra N, Li Z, Chen L, Li B, Zhang X, Wang C, Yeatman T, Coppola D and Chen J: SirT1 is an inhibitor of proliferation and tumor formation in colon cancer. J Biol Chem 284: 18210-18217, 2009.

13. Jung W, Hong KD, Jung WY, Lee E, Shin BK, Kim HK, Kim A and Kim BH: SIRT1 Expression Is Associated with Good Prognosis in Colorectal Cancer. Korean J Pathol 47: 332-339, 2013.

14. Kim JR, Moon YJ, Kwon KS, Bae JS, Wagle S, Yu TK, Kim KM, Park HS, Lee JH, Moon WS, et al: Expression of SIRT1 and $\mathrm{DBC} 1$ is associated with poor prognosis of soft tissue sarcomas. PLoS One 8: e74738, 2013

15. Leko V, Park G, Lao U, et al: Enterocyte-specific inactivation of SIRT1 reduces tumor load in the APC (+/min) mouse model. PLoS One 8: e66283, 2013.

16. Knight JR, Allison SJ and Milner J: Active regulator of SIRT1 is required for cancer cell survival but not for SIRT1 activity. Open Biol 3: 130-135, 2013.

17. Revollo JR and Li X: The ways and means that fine tune SIRT1 activity. Trends Biochem Sci 38: 160-167, 2013.

18. Zhang XL, Chen ML and Zhou SL: Fentanyl increases colorectal carcinoma cell apoptosis by inhibition of NF- $\kappa \mathrm{B}$ in a Sirt1-dependent manner. Asian Pac J Cancer Prev 15: 10015$10020,2014$.

19. The American Joint Committee on Cancer: The 7th edition of the AJCC cancer staging manual and the future of TNM. Ann Surg Oncol 17: 1471-1474, 2010.

20. Welch C, Chen Y and Stallings RL: MicroRNA-34a functions as a potential tumor suppressor by inducing apoptosis in neuroblastoma cells. Oncogene 26: 5017-5022, 2007.

21. Moretti RM, Montagnani Mareli M, Taylor DM, Martini PG, Marzagalli M and Limonta P: Gonadotropin-releasing hormone agonists sensitize and resensitize, prostate cancer cells to docetaxel in a p53-dependent manner. PLoS One 9: e93713, 2014.

22. Lodygin D, Tarasov V, Epanchintsev A, Berking C, Knyazeva T, Körner H, Knyazev P, Diebold J and Hermeking H: Inactivation of miR-34a by aberrant $\mathrm{CpG}$ methylation in multiple types of cancer. Cell Cycle 7: 2591-2600, 2008.

23. Yamakuchi M, Ferlito $M$ and Lowenstein CJ: MiR-34a repression of SIRT1 regulates apoptosis. Proc Natl Acad Sci U S A 105: 13421-13426, 2008.

24. Hwang BJ, Madabushi A, Jin J, Lin SY and Lu AL: Histone/protein deacetylase SIRT1 is an anticancer therapeutic target. Am J Cancer Res 4: 211-221, 2014.

25. Zhao T, Li J and Chen AF: MicroRNA-34a induces endothelial progenitor cell senescence and impedes its angiogenesis via suppressing silent information regulator 1. Am J Physiol Endocrinol Metab 299: E110-E116, 2010

26. Akao Y, Noguchi S, Iio A, Kojima K, Takagi T and Naoe T: Dysregulation of microR NA-34a expression causes drug-resistance to 5-FU in human colon cancer DLD-1 cells. Cancer Lett 300: 197-204, 2011.

27. Li L, Yuan L, Luo J, Gao J, Guo J and Xie X: MiR-34a inhibits proliferation and migration of breast cancer through down-regulation of Bcl-2 and SIRT1. Clin Exp Med 13: 109-117, 2013.

28. Chapman EJ, Kelly G and Knowles MA: Genes involved in differentiation, stem cell renewal and tumorigenesis are modulated in telomerase-immortalized human urothelial cells. Mol Cancer Res 6: 1154-1168, 2008.

29. Audrito V, Rossi D, Gottardi D, et al: Nicotinamide blocks proliferation and induces apoptosis of chronic lymphocytic leukemia cells through activation of the p53/miR-34a/SIRT1 tumor suppressor network. Cancer Res 71: 4473-4483, 2011.

30. Bommer GT, Feng Y, Kaczorowski AJ, Gerin I, Kuick R, Love RE, Zhai Y, Giordano TJ, et al: p53-mediated activation of miRNA34 candidate tumor-suppressor genes. Curr Biol 17: 1298-1307, 2007.

31. Chen F and Hu SJ: Effect of microRNA-34a in cell cycle, differentiation and apoptosis: A review. J Biochem Mol Toxicol 26: 79-86, 2012.

32. Ito T, Yagi S and Yamakuchi M: MicroRNA-34a regulation of endothelial senescence. Biochem Biophys Res Commun 398: 735-740, 2010. 